\title{
On the periodic solutions of a perturbed double pendulum
}

\section{J. Llibre}

Departament de Matematiques, Universitat Autònoma de Barcelona 08193 Bellaterra, Barcelona, Catalonia, Spain

E-mail address: jllibre@mat.uab.cat

\section{D. Novaes}

Departamento de Matematica, Universidade Estadual de Campinas Caixa Postal 6065, 13083-859, Campinas, SP, Brazil

E-mail address: ddnovaes@gmail.com

\section{A. Teixeira}

Departamento de Matematica, Universidade Estadual de Campinas Caixa Postal 6065, 13083-859, Campinas, SP, Brazil

E-mail address: teixeira@ime.unicamp.br

To Waldyr Oliva in his $80^{\text {th }}$ Birthday

\begin{abstract}
We provide sufficient conditions for the existence of periodic solutions of the planar perturbed double pendulum with small oscillations having equations of motion

$$
\begin{aligned}
& \ddot{\theta}_{1}=-2 a \theta_{1}+a \theta_{2}+\varepsilon F_{1}\left(t, \theta_{1}, \dot{\theta}_{1}, \theta_{2}, \dot{\theta}_{2}\right), \\
& \ddot{\theta}_{2}=2 a \theta_{1}-2 a \theta_{2}+\varepsilon F_{2}\left(t, \theta_{1}, \dot{\theta}_{1}, \theta_{2}, \dot{\theta}_{2}\right),
\end{aligned}
$$

where $a$ and $\varepsilon$ are real parameters. The two masses of the unperturbed double pendulum are equal, and its two stems have the same length $l$. In fact $a=g / l$ where $g$ is the acceleration of the gravity. Here the parameter $\varepsilon$ is small and the smooth functions $F_{1}$ and $F_{2}$ define the perturbation which are periodic functions in $t$ and in resonance $p: q$ with some of the periodic solutions of the unperturbed double pendulum, being $p$ and $q$ positive integers relatively prime.
\end{abstract}

2010 Mathematics Subject Classification. 37G15; 37C80; 37C30.

Key words: periodic solution, double pendulum, averaging theory. 


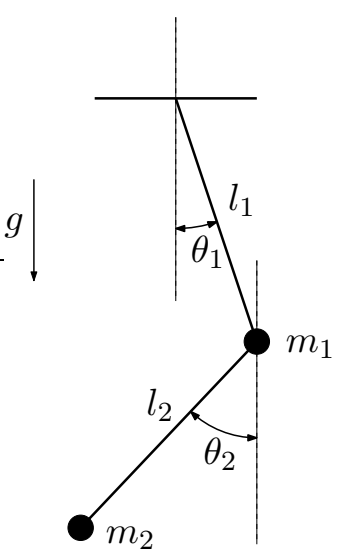

Figure 1. The planar double pendulum.

\section{Introduction and statement of the main results}

We consider a system of two point masses $m_{1}$ and $m_{2}$ moving in a fixed plane, in which the distance between a point (called pivot) and $m_{1}$ and the distance between $m_{1}$ and $m_{2}$ are fixed, and equal to $l_{1}$ and $l_{2}$ respectively. We assume the masses do not interact. We allow gravity to act on the masses $m_{1}$ and $m_{2}$. This system is called the planar double pendulum.

The position of the double pendulum is determined by the two angles $\theta_{1}$ and $\theta_{2}$ shown in Figure 1. We consider only the motion in the vicinity of the equilibrium $\theta_{1}=\theta_{2}=0$, i.e. we are only interested in small oscillations around this equilibrium. Expanding the Lagrangian of this system to second order in $\theta_{1}$ and $\theta_{2}$ and their time derivatives, the corresponding Lagrange equations of motion are

$$
\begin{aligned}
\left(m_{1}+m_{2}\right) l_{1} \ddot{\theta}_{1}+m_{2} l_{2} \ddot{\theta}_{2}+\left(m_{1}+m_{2}\right) g \theta_{1} & =0, \\
m_{2} l_{2} \ddot{\theta}_{2}+m_{2} l_{1} \ddot{\theta}_{1}+m_{2} g \theta_{2} & =0 .
\end{aligned}
$$

For more details on these equations of motion see [2]. Here the dot denotes derivative with respect to the time $t$.

If we take $m_{1}=m_{2}=m>0, l_{1}=l_{2}=l>0$ and divide the above equations by $m l$, system (1) becomes

$$
\begin{aligned}
2 \ddot{\theta}_{1}+\ddot{\theta}_{2}+2 \frac{g}{l} \theta_{1} & =0, \\
\ddot{\theta}_{1}+\ddot{\theta}_{2}+\frac{g}{l} \theta_{2} & =0 .
\end{aligned}
$$


Isolating $\ddot{\theta}_{1}$ and $\ddot{\theta}_{2}$ of equations (2) and denoting $a=g / l$, we obtain that the equations of motion of the double pendulum with small oscillations, two equal masses $m$ and having the two stems the same length $l$ are

$$
\begin{aligned}
& \ddot{\theta}_{1}=-2 a \theta_{1}+a \theta_{2}, \\
& \ddot{\theta}_{2}=2 a \theta_{1}-2 a \theta_{2},
\end{aligned}
$$

where $g$ is the acceleration of the gravity.

Taking a new time $\tau$ given by the rescaling $\tau=\sqrt{a} t$ the equations of motion (3) become

$$
\begin{aligned}
& \theta_{1}^{\prime \prime}=-2 \theta_{1}+\theta_{2}, \\
& \theta_{2}^{\prime \prime}=2 \theta_{1}-2 \theta_{2},
\end{aligned}
$$

where now the prime denotes derivative with respect to the new time $\tau$.

The objective of this paper is to provide a system of nonlinear equations whose simple zeros provide periodic solutions of the perturbed planar double pendulum with equations of motion

$$
\begin{aligned}
& \theta_{1}^{\prime \prime}=-2 \theta_{1}+\theta_{2}+\varepsilon F_{1}\left(\tau, \theta_{1}, \theta_{1}^{\prime}, \theta_{2}, \theta_{2}^{\prime}\right), \\
& \theta_{2}^{\prime \prime}=2 \theta_{1}-2 \theta_{2}+\varepsilon F_{2}\left(\tau, \theta_{1}, \theta_{1}^{\prime}, \theta_{2}, \theta_{2}^{\prime}\right),
\end{aligned}
$$

where $\varepsilon$ is a small parameter. Here the smooth functions $F_{1}$ and $F_{2}$ define the perturbation. These functions are periodic in $\tau$ and in resonance $p: q$ with some of the periodic solutions of the unperturbed double pendulum, being $p$ and $q$ positive integers relatively primes. In order to present our results we need some preliminary definitions and notations.

The unperturbed system (4) has a unique singular point, the origin with eigenvalues

$$
\pm \sqrt{2-\sqrt{2}} i, \quad \pm \sqrt{2+\sqrt{2}} i .
$$

Consequently this system in the phase space $\left(\theta_{1}, \theta_{1}^{\prime}, \theta_{2}, \theta_{2}^{\prime}\right)$ has two planes filled of periodic solutions with the exception of the origin. These periodic solutions have periods

$$
T_{1}=\frac{2 \pi}{\sqrt{2-\sqrt{2}}} \quad \text { or } \quad T_{2}=\frac{2 \pi}{\sqrt{2+\sqrt{2}}},
$$

according they belong to the plane associated to the eigenvectors with eigenvalues $\pm \sqrt{2-\sqrt{2}} i$ or $\pm \sqrt{2+\sqrt{2}} i$, respectively. We shall study which of these periodic solutions persist for the perturbed system (5) when the parameter $\varepsilon$ is sufficiently small and the perturbed functions $F_{i}$ for $i=1,2$ have period either $p T_{1} / q$, or $p T_{2} / q$, where $p$ and $q$ are positive integers relatively prime. 
We define the functions:

$$
\begin{aligned}
& \mathcal{G}_{1}\left(X_{0}, Y_{0}\right)=\int_{0}^{p T_{1}} \sin (\sqrt{2-\sqrt{2}} \tau)\left(\sqrt{2} \bar{F}_{1}+\bar{F}_{2}\right) d \tau \\
& \mathcal{G}_{2}\left(X_{0}, Y_{0}\right)=\int_{0}^{p T_{1}} \cos (\sqrt{2-\sqrt{2}} \tau)\left(\sqrt{2} \bar{F}_{1}+\bar{F}_{2}\right) d \tau
\end{aligned}
$$

where

$$
\bar{F}_{k}=F_{k}\left(\tau, A_{1}, B_{1}, C_{1}, D_{1}\right)
$$

for $k=1,2$ with

$$
\begin{aligned}
& A_{1}=\frac{1}{\sqrt{4-2 \sqrt{2}}}\left(X_{0} \cos (\sqrt{2-\sqrt{2}} \tau)+Y_{0} \sin (\sqrt{2-\sqrt{2}} \tau)\right), \\
& B_{1}=\frac{1}{\sqrt{2}}\left(Y_{0} \cos (\sqrt{2-\sqrt{2}} \tau)-X_{0} \sin (\sqrt{2-\sqrt{2}} \tau)\right) \\
& C_{1}=\frac{1}{\sqrt{2-\sqrt{2}}}\left(X_{0} \cos (\sqrt{2-\sqrt{2}} \tau)+Y_{0} \sin (\sqrt{2-\sqrt{2}} \tau)\right), \\
& D_{1}=Y_{0} \cos (\sqrt{2-\sqrt{2}} \tau)-X_{0} \sin (\sqrt{2-\sqrt{2}} \tau) .
\end{aligned}
$$

A zero $\left(X_{0}^{*}, Y_{0}^{*}\right)$ of the nonlinear system

$$
\mathcal{G}_{1}\left(X_{0}, Y_{0}\right)=0, \quad \mathcal{G}_{2}\left(X_{0}, Y_{0}\right)=0,
$$

such that

$$
\operatorname{det}\left(\left.\frac{\partial\left(\mathcal{G}_{1}, \mathcal{G}_{2}\right)}{\partial\left(X_{0}, Y_{0}\right)}\right|_{\left(X_{0}, Y_{0}\right)=\left(X_{0}^{*}, Y_{0}^{*}\right)}\right) \neq 0,
$$

is called a simple zero of system (8).

Our main result on the periodic solutions of the perturbed double pendulum (5) which bifurcate from the periodic solutions of the unperturbed double pendulum (4) with period $T_{1}$ traveled $p$ times is the following.

Theorem 1. Assume that the functions $F_{k}$ of the perturbed double pendulum with equations of motion (5) are periodic in $\tau$ of period $p T_{1} / q$ with $p$ and $q$ positive integers relatively prime. Then for $\varepsilon \neq 0$ sufficiently small and for every simple zero $\left(X_{0}^{*}, Y_{0}^{*}\right) \neq(0,0)$ of the nonlinear system (8), the perturbed double pendulum (5) has a periodic solution $\left(\theta_{1}(\tau, \varepsilon), \theta_{2}(\tau, \varepsilon)\right)$ tending when $\varepsilon \rightarrow 0$ to the periodic solution $\left(\theta_{1}(\tau), \theta_{2}(\tau)\right)$ run $p$ times of 
the unperturbed double pendulum (4) given by

$$
\begin{aligned}
& \left(\frac{1}{\sqrt{4-2 \sqrt{2}}}\left(X_{0}^{*} \cos (\sqrt{2-\sqrt{2}} \tau)+Y_{0}^{*} \sin (\sqrt{2-\sqrt{2}} \tau)\right),\right. \\
& \left.\frac{1}{\sqrt{2-\sqrt{2}}}\left(X_{0}^{*} \cos (\sqrt{2-\sqrt{2}} \tau)+Y_{0}^{*} \sin (\sqrt{2-\sqrt{2}} \tau)\right)\right) .
\end{aligned}
$$

Theorem 1 is proved in section 2. Its proof is based in the averaging theory for computing periodic solutions, see the appendix.

We provide an application of Theorem 1 in the following corollary, which will be proved in section 3 .

Corollary 2. If $F_{2}\left(\theta_{1}, \theta_{1}^{\prime}, \theta_{2}, \theta_{2}^{\prime}\right)=\left(1-\theta_{1}^{2}\right) \sin (\sqrt{2-\sqrt{2}} t)$ and $F_{1}\left(\theta_{1}, \theta_{1}^{\prime}, \theta_{2}, \theta_{2}^{\prime}\right)=0$, then the differential equation (5) for $\varepsilon \neq 0$ sufficiently small has two periodic solutions $\left(\theta_{1}(\tau, \varepsilon), \theta_{2}(\tau, \varepsilon)\right)$ tending when $\varepsilon \rightarrow 0$ to the two periodic solutions $\left(\theta_{1}(\tau), \theta_{2}(\tau)\right)$ of the unperturbed double pendulum (4) given by (9) with

$$
\left(X_{0}^{*}, Y_{0}^{*}\right)=(2 \sqrt{2(2-\sqrt{2})}, 0) \text { and }\left(X_{0}^{*}, Y_{0}^{*}\right)=\left(0,2 \sqrt{\frac{2}{3}(2-\sqrt{2})}\right),
$$

respectively.

Now we define the functions:

$$
\begin{aligned}
& \mathcal{G}^{1}\left(Z_{0}, W_{0}\right)=\int_{0}^{p T_{2}} \sin (\sqrt{2+\sqrt{2}} \tau)\left(-\sqrt{2} \bar{F}_{1}+\bar{F}_{2}\right) d \tau, \\
& \mathcal{G}^{2}\left(Z_{0}, W_{0}\right)=\int_{0}^{p T_{2}} \cos (\sqrt{2+\sqrt{2}} \tau)\left(-\sqrt{2} \bar{F}_{1}+\bar{F}_{2}\right) d \tau,
\end{aligned}
$$

where $\bar{F}_{k}=F_{k}\left(\tau, A_{2}, B_{2}, C_{2}, D_{2}\right)$ for $k=1,2$ with

$$
\begin{aligned}
& A_{2}=-\frac{1}{\sqrt{4+2 \sqrt{2}}}\left(Z_{0} \cos (\sqrt{2+\sqrt{2}} \tau)+W_{0} \sin (\sqrt{2+\sqrt{2}} \tau)\right), \\
& B_{2}=\frac{1}{\sqrt{2}}\left(-W_{0} \cos (\sqrt{2+\sqrt{2}} \tau)+Z_{0} \sin (\sqrt{2+\sqrt{2}} \tau)\right), \\
& C_{2}=\frac{1}{\sqrt{2+\sqrt{2}}}\left(Z_{0} \cos (\sqrt{2+\sqrt{2}} \tau)+W_{0} \sin (\sqrt{2+\sqrt{2}} \tau)\right), \\
& D_{2}=W_{0} \cos (\sqrt{2+\sqrt{2}} \tau)-Z_{0} \sin (\sqrt{2+\sqrt{2}} \tau) .
\end{aligned}
$$


Consider the nonlinear system

$$
\mathcal{G}^{1}\left(Z_{0}, W_{0}\right)=0, \quad \mathcal{G}^{2}\left(Z_{0}, W_{0}\right)=0 .
$$

Our main result on the periodic solutions of the perturbed double pendulum (5) which bifurcate from the periodic solutions of the unperturbed double pendulum (4) with period $T_{2}$ traveled $p$ times is the following.

Theorem 3. Assume that the functions $F_{k}$ of the perturbed double pendulum with equations of motion (5) are periodic in $\tau$ of period $p T_{2} / q$ with $p$ and $q$ positive integers relatively prime. Then for $\varepsilon \neq 0$ sufficiently small and for every simple zero $\left(Z_{0}^{*}, W_{0}^{*}\right) \neq(0,0)$ of the nonlinear system (11), the perturbed double pendulum (5) has a periodic solution $\left(\theta_{1}(\tau, \varepsilon), \theta_{2}(\tau, \varepsilon)\right)$ tending when $\varepsilon \rightarrow 0$ to the periodic solution $\left(\theta_{1}(\tau), \theta_{2}(\tau)\right)$ run $p$ times of the unperturbed double pendulum (4) given by

$$
\begin{aligned}
& \left(-\frac{1}{\sqrt{4+2 \sqrt{2}}}\left(Z_{0}^{*} \cos (\sqrt{2+\sqrt{2}} \tau)+W_{0}^{*} \sin (\sqrt{2+\sqrt{2}} \tau)\right),\right. \\
& \left.\frac{1}{\sqrt{2+\sqrt{2}}}\left(Z_{0}^{*} \cos (\sqrt{2+\sqrt{2}} \tau)+W_{0}^{*} \sin (\sqrt{2+\sqrt{2}} \tau)\right)\right) .
\end{aligned}
$$

Theorem 3 is also proved in section 2 .

Now we provide an application of Theorem 3 in the next corollary, which will be proved in section 3 .

Corollary 4. If $F_{1}\left(\theta_{1}, \theta_{1}^{\prime}, \theta_{2}, \theta_{2}^{\prime}\right)=\theta_{2}^{\prime}+\theta_{1}^{2} \cos (\sqrt{2+\sqrt{2}} t)$ and $F_{2}\left(\theta_{1}, \theta_{1}^{\prime}, \theta_{2}, \theta_{2}^{\prime}\right)=0$, then the differential equation (5) for $\varepsilon \neq 0$ sufficiently small has one periodic solution $\left(\theta_{1}(\tau, \varepsilon), \theta_{2}(\tau, \varepsilon)\right)$ tending when $\varepsilon \rightarrow 0$ to the periodic solution $\left(\theta_{1}(\tau), \theta_{2}(\tau)\right)$ of the unperturbed double pendulum (4) given by $(12)$ with $\left(Z_{0}^{*}, W_{0}^{*}\right)=(0,-8(2+\sqrt{2}))$.

\section{Proof of Theorems 1 and 3}

Introducing the variables $(x, y, z, w)=\left(\theta_{1}, \theta_{1}^{\prime}, \theta_{2}, \theta_{2}^{\prime}\right)$ we write the differential system of the perturbed double pendulum (5) as a first-order differential system defined in $\mathbb{R}^{4}$. Thus we have the differential system

$$
\begin{aligned}
& x^{\prime}=y, \\
& y^{\prime}=-2 x+z+\varepsilon F_{1}(\tau, x, y, z, w), \\
& z^{\prime}=w, \\
& w^{\prime}=2 x-2 z+\varepsilon F_{2}(\tau, x, y, z, w) .
\end{aligned}
$$


System (13) with $\varepsilon=0$ is equivalent to the unperturbed double pendulum system (4), called in what follows simply by the unperturbed system, otherwise we have the perturbed system.

We shall write system (13) in such a way that the linear part at the origin will be in its real normal Jordan form. Then, doing the change of variables $(\tau, x, y, z, w) \rightarrow(\tau, X, Y, Z, W)$ given by

$$
\left(\begin{array}{c}
X \\
Y \\
Z \\
W
\end{array}\right)=\left(\begin{array}{cccc}
\sqrt{1-\frac{1}{\sqrt{2}}} & 0 & \frac{\sqrt{2-\sqrt{2}}}{2} & 0 \\
0 & \frac{1}{\sqrt{2}} & 0 & \frac{1}{2} \\
-\sqrt{1+\frac{1}{\sqrt{2}}} & 0 & \frac{\sqrt{2+\sqrt{2}}}{2} & 0 \\
0 & -\frac{1}{\sqrt{2}} & 0 & \frac{1}{2}
\end{array}\right)\left(\begin{array}{c}
x \\
y \\
z \\
w
\end{array}\right),
$$

the differential system (13) becomes

$$
\begin{aligned}
& X^{\prime}=\sqrt{2-\sqrt{2}} Y, \\
& Y^{\prime}=-\sqrt{2-\sqrt{2}} X+\varepsilon \frac{1}{2}\left(\sqrt{2} \tilde{F}_{1}+\tilde{F}_{2}\right), \\
& Z^{\prime}=\sqrt{2+\sqrt{2}} W \\
& W^{\prime}=-\sqrt{2+\sqrt{2}} Z+\varepsilon \frac{1}{2}\left(\tilde{F}_{2}-\sqrt{2} \tilde{F}_{1}\right),
\end{aligned}
$$

where $\tilde{F}_{i}(\tau, X, Y, Z, W)=F_{i}(\tau, \mathcal{A}, \mathcal{B}, \mathcal{C}, \mathcal{D})$ for $i=1,2$ with

$$
\begin{aligned}
& \mathcal{A}=\frac{X}{\sqrt{4-2 \sqrt{2}}}-\frac{Z}{\sqrt{2(2+\sqrt{2})}}, \\
& \mathcal{B}=\frac{Y-W}{\sqrt{2}} \\
& \mathcal{C}=\frac{X}{\sqrt{2-\sqrt{2}}}+\frac{Z}{\sqrt{2+\sqrt{2}}}, \\
& \mathcal{D}=Y+W .
\end{aligned}
$$

Note that the linear part of the differential system (15) at the origin is in its real normal Jordan form. 
Lemma 5. The periodic solutions of the differential system (15) with $\varepsilon=0$ are

$$
\begin{aligned}
& X(\tau)=X_{0} \cos (\sqrt{2-\sqrt{2}} \tau)+Y_{0} \sin (\sqrt{2-\sqrt{2}} \tau), \\
& Y(\tau)=Y_{0} \cos (\sqrt{2-\sqrt{2}} \tau)-X_{0} \sin (\sqrt{2-\sqrt{2}} \tau), \\
& Z(\tau)=0, \\
& W(\tau)=0,
\end{aligned}
$$

of period $T_{1}$, and

$$
\begin{aligned}
& X(\tau)=0 \\
& Y(\tau)=0 \\
& Z(\tau)=Z_{0} \cos (\sqrt{2+\sqrt{2}} \tau)+W_{0} \sin (\sqrt{2+\sqrt{2}} \tau), \\
& W(\tau)=W_{0} \cos (\sqrt{2+\sqrt{2}} \tau)-Z_{0} \sin (\sqrt{2+\sqrt{2}} \tau),
\end{aligned}
$$

of period $T_{2}$.

Proof. Since system (15) with $\varepsilon=0$ is a linear differential system, the proof follows easily.

Proof of Theorem 1. Assume that the functions $F_{k}$ of the perturbed double pendulum with equations of motion (5) are periodic in $\tau$ of period $p T_{1} / q$ with $p$ and $q$ positive integers relatively prime. Then we can think that system (5) is periodic in $\tau$ of period $p T_{1}$. Thinking in this way the differential system and the periodic solutions (16) have the same period $p T_{1}$.

We shall apply Theorem 6 of the appendix to the differential system (15). We note that system (15) can be written as system (19) taking

$$
\begin{gathered}
\mathbf{x}=\left(\begin{array}{c}
X \\
Y \\
Z \\
W
\end{array}\right), \quad t=\tau, \quad G_{0}(t, \mathbf{x})=\left(\begin{array}{c}
\sqrt{2-\sqrt{2}} Y, \\
-\sqrt{2-\sqrt{2}} X, \\
\sqrt{2+\sqrt{2}} W, \\
-\sqrt{2+\sqrt{2}} Z
\end{array}\right), \\
G_{1}(t, \mathbf{x})=\left(\begin{array}{c}
0 \\
\frac{1}{2}\left(\sqrt{2} \tilde{F}_{1}+\tilde{F}_{2}\right) \\
0 \\
\frac{1}{2}\left(\tilde{F}_{2}-\sqrt{2} \tilde{F}_{1}\right)
\end{array}\right) \text { and } G_{2}(t, \mathbf{x}, \varepsilon)=\left(\begin{array}{l}
0 \\
0 \\
0 \\
0
\end{array}\right) .
\end{gathered}
$$


We shall study which periodic solutions (16) of the unperturbed system (15) with $\varepsilon=0$ can be continued to periodic solutions of the unperturbed system (15) for $\varepsilon \neq 0$ sufficiently small.

We shall describe the different elements which appear in the statement of Theorem 6 in the particular case of the differential system (15). Thus we have that $\Omega=\mathbb{R}^{4}, k=2$ and $n=4$. Let $r_{1}>0$ be arbitrarily small and let $r_{2}>0$ be arbitrarily large. We take the open and bounded subset $V$ of the plane $Z=W=0$ as

$$
V=\left\{\left(X_{0}, Y_{0}, 0,0\right) \in \mathbb{R}^{4}: r_{1}<\sqrt{X_{0}^{2}+Y_{0}^{2}}<r_{2}\right\} .
$$

As usual $\mathrm{Cl}(V)$ denotes the closure of $V$. If $\alpha=\left(X_{0}, Y_{0}\right)$, then we can identify $V$ with the set

$$
\left\{\alpha \in \mathbb{R}^{2}: r_{1}<\|\alpha\|<r_{2}\right\},
$$

here $\|\cdot\|$ denotes the Euclidean norm of $\mathbb{R}^{2}$. The function $\beta: \operatorname{Cl}(V) \rightarrow \mathbb{R}^{2}$ is $\beta(\alpha)=(0,0)$. Therefore, in our case the set

$$
\begin{aligned}
\mathcal{Z} & =\left\{\mathbf{z}_{\alpha}=(\alpha, \beta(\alpha)), \alpha \in \mathrm{Cl}(V)\right\} \\
& =\left\{\left(X_{0}, Y_{0}, 0,0\right) \in \mathbb{R}^{4}: r_{1} \leq \sqrt{X_{0}^{2}+Y_{0}^{2}} \leq r_{2}\right\} .
\end{aligned}
$$

Clearly for each $\mathbf{z}_{\alpha} \in \mathcal{Z}$ we can consider the periodic solution $\mathbf{x}\left(\tau, \mathbf{z}_{\alpha}\right)=$ $(X(\tau), Y(\tau), 0,0)$ given by (16) of period $p T_{1}$.

Computing the fundamental matrix $M_{\mathbf{z}_{\alpha}}(\tau)$ of the linear differential system (15) with $\varepsilon=0$ associated to the $T$-periodic solution $\mathbf{z}_{\alpha}=\left(X_{0}, Y_{0}, 0,0\right)$ such that $M_{\mathbf{z}_{\alpha}}(0)$ be the identity of $\mathbb{R}^{4}$, we get that $M(\tau)=M_{\mathbf{z}_{\alpha}}(\tau)$ is equal to

$$
\left(\begin{array}{cccc}
\cos (\sqrt{2-\sqrt{2}} \tau) & \sin (\sqrt{2-\sqrt{2}} \tau) & 0 & 0 \\
-\sin (\sqrt{2-\sqrt{2}} \tau) & \cos (\sqrt{2-\sqrt{2}} \tau) & 0 & 0 \\
0 & 0 & \cos (\sqrt{2+\sqrt{2}} \tau) & \sin (\sqrt{2+\sqrt{2}} \tau) \\
0 & 0 & -\sin (\sqrt{2+\sqrt{2}}) & \cos (\sqrt{2+\sqrt{2}} \tau)
\end{array}\right)
$$

Note that the matrix $M_{\mathbf{z}_{\alpha}}(\tau)$ does not depend of the particular periodic solution $\mathbf{x}\left(\tau, \mathbf{z}_{\alpha}\right)$. Since the matrix

$$
M^{-1}(0)-M^{-1}\left(p T_{1}\right)=\left(\begin{array}{cccc}
0 & 0 & 0 & 0 \\
0 & 0 & 0 & 0 \\
0 & 0 & 2 \sin ^{2}(\sqrt{2} \pi) & \sin (2 \sqrt{2} \pi) \\
0 & 0 & -\sin (2 \sqrt{2} \pi) & 2 \sin ^{2}(\sqrt{2} \pi)
\end{array}\right)
$$


satisfies the assumptions of statement (ii) of Theorem 6 because the determinant

$$
\left|\begin{array}{cc}
2 \sin ^{2}(\sqrt{2} \pi) & \sin (2 \sqrt{2} \pi) \\
-\sin (2 \sqrt{2} \pi) & 2 \sin ^{2}(\sqrt{2} \pi)
\end{array}\right|=4 \sin ^{2}(\sqrt{2} \pi) \neq 0,
$$

we can apply this theorem to system (15).

Now $\xi: \mathbb{R}^{4} \rightarrow \mathbb{R}^{2}$ is $\xi(X, Y, Z, W)=(X, Y)$. We calculate the function

$$
\mathcal{G}\left(X_{0}, Y_{0}\right)=\mathcal{G}(\alpha)=\xi\left(\frac{1}{p T_{1}} \int_{0}^{p T_{1}} M_{\mathbf{z}_{\alpha}}^{-1}(\tau) G_{1}\left(\tau, \mathbf{x}\left(\tau, \mathbf{z}_{\alpha}\right)\right) d \tau\right),
$$

and we obtain

$$
\left(\begin{array}{l}
\mathcal{G}_{1}\left(X_{0}, Y_{0}\right) \\
\mathcal{G}_{2}\left(X_{0}, Y_{0}\right)
\end{array}\right)=\left(\begin{array}{c}
-\frac{\sqrt{2-\sqrt{2}}}{4 \pi} \int_{0}^{p T_{1}} \sin (\sqrt{2-\sqrt{2}} \tau)\left(\sqrt{2} \bar{F}_{1}+\bar{F}_{2}\right) d \tau \\
\frac{\sqrt{2-\sqrt{2}}}{4 \pi} \int_{0}^{p T_{1}} \cos (\sqrt{2-\sqrt{2}} \tau)\left(\sqrt{2} \bar{F}_{1}+\bar{F}_{2}\right) d \tau
\end{array}\right)
$$

where the functions of $\bar{F}_{k}$ for $k=1,2$ are the ones given in (7). Then, by Theorem 6 we have that for every simple zero $\left(X_{0}^{*}, Y_{0}^{*}\right) \in V$ of the system of nonlinear functions

$$
\mathcal{G}_{1}\left(X_{0}, Y_{0}\right)=0, \quad \mathcal{G}_{2}\left(X_{0}, Y_{0}\right)=0,
$$

we have a periodic solution $(X, Y, Z, W)(\tau, \varepsilon)$ of system (15) such that

$$
(X, Y, Z, W)(\tau, \varepsilon) \rightarrow\left(X_{0}^{*}, Y_{0}^{*}, 0,0\right) \text { as } \varepsilon \rightarrow 0 .
$$

Note that system (18) is equivalent to system (8), because both equations only differs in a non-zero multiplicative constant.

Going back through the change of coordinates (14) we get a periodic solution $(x, y, z, w)(\tau, \varepsilon)$ of system (16) such that

$$
\left(\begin{array}{c}
x(\tau, \varepsilon) \\
y(\tau, \varepsilon) \\
z(\tau, \varepsilon) \\
w(\tau, \varepsilon)
\end{array}\right) \rightarrow\left(\begin{array}{c}
\frac{1}{\sqrt{4-2 \sqrt{2}}}\left(X_{0}^{*} \cos (\sqrt{2-\sqrt{2}} \tau)+Y_{0}^{*} \sin (\sqrt{2-\sqrt{2}} \tau)\right) \\
\frac{1}{\sqrt{2}}\left(Y_{0}^{*} \cos (\sqrt{2-\sqrt{2}} \tau)-X_{0}^{*} \sin (\sqrt{2-\sqrt{2}} \tau)\right) \\
\frac{1}{\sqrt{2-\sqrt{2}}}\left(X_{0}^{*} \cos (\sqrt{2-\sqrt{2}} \tau)+Y_{0}^{*} \sin (\sqrt{2-\sqrt{2}} \tau)\right) \\
Y_{0}^{*} \cos (\sqrt{2-\sqrt{2}} \tau)-X_{0}^{*} \sin (\sqrt{2-\sqrt{2}} \tau)
\end{array}\right)
$$

as $\varepsilon \rightarrow 0$. 
Consequently we obtain a periodic solution $\left(\theta_{1}, \theta_{2}\right)(\tau, \varepsilon)$ of system (5) such that

$\left(\theta_{1}, \theta_{2}\right)(\tau, \varepsilon) \rightarrow\left(\begin{array}{l}\frac{1}{\sqrt{4-2 \sqrt{2}}}\left(X_{0}^{*} \cos (\sqrt{2-\sqrt{2}} \tau)+Y_{0}^{*} \sin (\sqrt{2-\sqrt{2}} \tau)\right) \\ \frac{1}{\sqrt{2-\sqrt{2}}}\left(X_{0}^{*} \cos (\sqrt{2-\sqrt{2}} \tau)+Y_{0}^{*} \sin (\sqrt{2-\sqrt{2}} \tau)\right)\end{array}\right)$

as $\varepsilon \rightarrow 0$. Hence Theorem 1 is proved.

Proof of Theorem 3. This proof is completely analogous to the proof of Theorem 1.

\section{Proof of the two corollaries}

Proof of Corollary 2. Under the assumptions of Corollary 2 the nonlinear system (6) becomes

$$
\begin{aligned}
& \mathcal{G}_{1}\left(X_{0}, Y_{0}\right)=-\frac{\pi\left(X_{0}^{2}+3 Y_{0}^{2}+8(-2+\sqrt{2})\right)}{8(2-\sqrt{2})^{3 / 2}}, \\
& \mathcal{G}_{2}\left(X_{0}, Y_{0}\right)=-\frac{\pi X_{0} Y_{0}}{4(2-\sqrt{2})^{3 / 2}} .
\end{aligned}
$$

This system has the following four solutions

$$
\left(X_{0}^{*}, Y_{0}^{*}\right)=( \pm 2 \sqrt{2(2-\sqrt{2})}, 0) \text { and }\left(X_{0}^{*}, Y_{0}^{*}\right)=\left(0, \pm 2 \sqrt{\frac{2}{3}(2-\sqrt{2})}\right) .
$$

But the solutions which differs in a sign are different initial conditions of the same periodic solution of the unperturbed double pendulum. Moreover, it easy to check that these solutions are simple. So, by Theorem 1 we only have two periodic solutions of the perturbed double pendulum. This completes the proof of the corollary. 
Proof of Corollary 4. Under the assumptions of Corollary 4 the nonlinear system (10) becomes

$$
\begin{aligned}
\mathcal{G}^{1}\left(Z_{0}, W_{0}\right) & =-\frac{\pi\left(\sqrt{(10-7 \sqrt{2})(2+\sqrt{2})} W_{0}-8\right) Z_{0}}{4 \sqrt{2(2+\sqrt{2})}}, \\
\mathcal{G}^{2}\left(Z_{0}, W_{0}\right) & =\frac{\pi\left(\sqrt{2} W_{0}^{2}-2 W_{0}^{2}-16 W_{0}+3 \sqrt{2} Z_{0}^{2}-6 Z_{0}^{2}\right)}{8 \sqrt{2(2+\sqrt{2})}} .
\end{aligned}
$$

This system only has the following two real solutions

$$
\left(\left(Z_{0}^{*}, W_{0}^{*}\right)=(0,-8(2+\sqrt{2})) \text { and }\left(Z_{0}^{*}, W_{0}^{*}\right)=(0,0) .\right.
$$

the other two solutions are not real. But the solution $(0,0)$ is not valid. Therefore, by Theorem 3 we only have one periodic solution of the perturbed double pendulum. This completes the proof of the corollary.

\section{Appendix: Basic results on averaging theory}

In this appendix we present the basic result from the averaging theory that we shall need for proving the main results of this paper.

We consider the problem of the bifurcation of $T$-periodic solutions from differential systems of the form

$$
\dot{\mathbf{x}}(t)=G_{0}(t, \mathbf{x})+\varepsilon G_{1}(t, \mathbf{x})+\varepsilon^{2} G_{2}(t, \mathbf{x}, \varepsilon),
$$

with $\varepsilon=0$ to $\varepsilon \neq 0$ sufficiently small. Here the functions $G_{0}, G_{1}: \mathbb{R} \times \Omega \rightarrow$ $\mathbb{R}^{n}$ and $G_{2}: \mathbb{R} \times \Omega \times\left(-\varepsilon_{0}, \varepsilon_{0}\right) \rightarrow \mathbb{R}^{n}$ are $\mathcal{C}^{2}$ functions, $T$-periodic in the first variable, and $\Omega$ is an open subset of $\mathbb{R}^{n}$. The main assumption is that the unperturbed system

$$
\dot{\mathbf{x}}(t)=G_{0}(t, \mathbf{x}),
$$

has a submanifold of periodic solutions. A solution of this problem is given using the averaging theory.

Let $\mathbf{x}(t, \mathbf{z}, \varepsilon)$ be the solution of the system (20) such that $\mathbf{x}(0, \mathbf{z}, \varepsilon)=$ $\mathbf{z}$. We write the linearization of the unperturbed system along a periodic solution $\mathbf{x}(t, \mathbf{z}, 0)$ as

$$
\dot{\mathbf{y}}=D_{\mathbf{x}} G_{0}(t, \mathbf{x}(t, \mathbf{z}, 0)) \mathbf{y} .
$$

In what follows we denote by $M_{\mathbf{z}}(t)$ some fundamental matrix of the linear differential system $(21)$, and by $\xi: \mathbb{R}^{k} \times \mathbb{R}^{n-k} \rightarrow \mathbb{R}^{k}$ the projection of $\mathbb{R}^{n}$ onto its first $k$ coordinates; i.e. $\xi\left(x_{1}, \ldots, x_{n}\right)=\left(x_{1}, \ldots, x_{k}\right)$. 
We assume that there exists a $k$-dimensional submanifold $\mathcal{Z}$ of $\Omega$ filled with $T$-periodic solutions of (20). Then an answer to the problem of bifurcation of $T$-periodic solutions from the periodic solutions contained in $\mathcal{Z}$ for system (19) is given in the following result.

Theorem 6. Let $V$ be an open and bounded subset of $\mathbb{R}^{k}$, and let $\beta$ : $\mathrm{Cl}(V) \rightarrow \mathbb{R}^{n-k}$ be a $\mathcal{C}^{2}$ function. We assume that

(i) $\mathcal{Z}=\left\{\mathbf{z}_{\alpha}=(\alpha, \beta(\alpha)), \alpha \in \mathrm{Cl}(V)\right\} \subset \Omega$ and that for each $\mathbf{z}_{\alpha} \in \mathcal{Z}$ the solution $\mathbf{x}\left(t, \mathbf{z}_{\alpha}\right)$ of (20) is T-periodic;

(ii) for each $\mathbf{z}_{\alpha} \in \mathcal{Z}$ there is a fundamental matrix $M_{\mathbf{z}_{\alpha}}(t)$ of (21) such that the matrix $M_{\mathbf{z}_{\alpha}}^{-1}(0)-M_{\mathbf{z}_{\alpha}}^{-1}(T)$ has in the upper right corner the $k \times(n-k)$ zero matrix, and in the lower right corner a $(n-k) \times(n-k)$ matrix $\Delta_{\alpha}$ with $\operatorname{det}\left(\Delta_{\alpha}\right) \neq 0$.

We consider the function $\mathcal{G}: \mathrm{Cl}(V) \rightarrow \mathbb{R}^{k}$

$$
\mathcal{G}(\alpha)=\xi\left(\frac{1}{T} \int_{0}^{T} M_{\mathbf{z}_{\alpha}}^{-1}(t) G_{1}\left(t, \mathbf{x}\left(t, \mathbf{z}_{\alpha}\right)\right) d t\right) .
$$

If there exists $a \in V$ with $\mathcal{G}(a)=0$ and $\operatorname{det}((d \mathcal{G} / d \alpha)(a)) \neq 0$, then there is a T-periodic solution $\varphi(t, \varepsilon)$ of system (19) such that $\varphi(0, \varepsilon) \rightarrow \mathbf{z}_{a}$ as $\varepsilon \rightarrow 0$.

Theorem 6 goes back to Malkin [3] and Roseau [4], for a shorter proof see $[1]$.

\section{Acknowledgements}

The first author is partially supported by a MICINN/FEDER grant MTM2008-03437, by a AGAUR grant number 2009SGR-0410 and by ICREA Academia. The second author is partially suported by the grant FAPESP 2011/03896-0 The third author is partially supported by a FAPESP-BRAZIL grant 2007/06896-5. The first and third authors are also supported by the joint project CAPES-MECD grant PHB-2009-0025$\mathrm{PC}$

\section{References}

[1] A. Buică, J.P. Françoise And J. Llibre, Periodic solutions of nonlinear periodic differential systems with a small parameter, Communication on Pure and Applied Analysis 6 (2007), 103-111.

[2] H. IRo, A modern approach to classical mechanics, World Scientific Publishing Co., Inc., River Edge, NJ, 2002.

[3] I.G. MaLkin, Some problems of the theory of nonlinear oscillations, (Russian) Gosudarstv. Izdat. Tehn.-Teor. Lit., Moscow, 1956. 
[4] M. Roseau, Vibrations non linéaires et théorie de la stabilité, (French) Springer Tracts in Natural Philosophy, Vol.8 Springer-Verlag, Berlin-New York, 1966.

São Paulo J.Math.Sci. 5, 2 (2011), 317-330 